\title{
CONTINUOUS-TIME ADAPTIVE CONTROL APPLIED TO RF AMPLIFIER LINEARIZATION
}

Bertran, E.: Montoro, G.: Talavera, N.

Department of Signal Theory and Communications

Universitat Politècnica de Catalunya

PO. BOX: 30002 08080- BARCELONA (SPAIN)

FAX: + 3434015910

Abstract.- A new approach to the RF power amplifier linearization problem is presented. The proposed solution apply non-linear theories (Lyapunov direct method) to adaptive filtering in order to improve the linearity of the RF amplifiers. The obtained design requires lower circuit complexity than the LINC amplifier, and is not based on iterative algorithms nor sub-system identification. Up to $100 \mathrm{MHz}$ these functions could be implemented, at present, with operational amplifiers and integrated analog multipliers (four quadrants). The adjusting algorithm convergence or the interruption of the communication are not problems in the proposed adaptive solution.

The canceller structure design is based on model reference adaptive systems (MRAS): to cancel the error between the plant output (distortioned output of the RF amplifier) and reference model (the desired signal obtained from a linear and low-power amplifier) by using continuous-time techniques.

The proposed structure is experimented by computer simulation (SPICE program) in a class-A RF power amplifier. The behaviour of the adapted amplifier is studied when power transistors approaches nonlinear operating zones (saturation state).

\section{INTRODUCTION}

Linear RF power amplifiers used in mobile radio systems require good power efficiency and low out-of-band emission. To achieve these requirements it is necessary to reduce nonlinearities in RF power stages, and different techniques have been used to solve this problem. Apart from the use of several power transistors operating in parallel, the classical way is to apply conventional (cartesian) feedback, a not expensive solution with stability and delay compensation problems. So, the amplifier band-width is reduced in order to preserve phasemargin in the closed-loop amplifier, and the robustness is low [1].

Recently signal processing techniques have been used, as is the case of adaptive predistortion and others based on the feedforward of the distortioned output signal [2], [3]. The main restriction in adaptive predistortion is the interruption of the communication during the time intervals necessary to adjust the predistorter parameters or to update the look-up tables according to transmitter non-linearities. If the predistortion technique is used without adaptive possibilities, the feedforwated harmonics to be subtracted to those generated by the power amplifier must be phase-compensated. Other important signal processing technique is the LINC amplifier, that produces linear amplification of bandpass signals with nonlinear components by forming two constant envelope phase inodulated components from the bandpass input and recombining the amplified components [4]. This amplifier achieves high efficiency and spurious are reduced in the order of $30-60 \mathrm{dBc}$. Its limitation is the sensitivity of the design to the balance between the two amplifiers of each component of the bandpass input signal. More advanced techniques try to apply Volterra series or neural-nets to predistortion power amplifiers [5], [6].

Some adaptive predistortion methods are supported by Digital Signal Processor (DSP) based designs, with restrictions due to the required computing-time and $A / D$ and $D / A$ converters (price, conversion time and quantization noise). The computingtime restriction can be relaxed with a translation to base-band of the numerical operations, nevertheless the operating capacity is around $100-200 \mathrm{KHz}$.

Some techniques used to minimize the distortion caused by the RF power amplifiers have its origin in the field of Control Theory (recursive identification, adaptive control). The closedloop systems used to compensate, non-linearities can be considered as a feedback system with a linear path and another non-linear [7]. Adaptive Filtering and Control theories have been well exploited in Communications. especially those based on the previous identification of some sub-system characteristics (by sending test pulses or pseudo-random sequences), or based on the adjustment of parameters after certain number of iterations [8], [9]. Both methodologies requires a DSP-based board to be implemented. Other methodologies of design, as the continuous-time model reference adaptive systems (MRAS), based on non-linear systems stability [10] have been seldom exported to applications in Communications, in spite that they are well structured in the Control Theory field.

The linearization of the RF power amplifier, with the consequent spectral efficiency, can be improved either by designing in the frequency domain or in the time domain. In this paper a new time domain approach to the RF power amplifier linearization problem is presented.

The proposed solution applies non-linear theories (Lyapunov methods) to adaptive filtering in order to improve the linearity of the RF amplifiers. The adjusting algorithm convergence or the interruption of the communication are not problems in the proposed adaptive solution. The obtained design requires lower circuit complexity than others alternatives, and it is not based on iterative algorithms nor sub-system identification. 
The structure is the basic one in model reference adaptive systems: to cancel the error signal between the distortioned output of the RF amplifier and the desired signal obtained from a linear and low-power reference model (the comparison is carried out in terms of continuous-time output voltage). The method here used to cancel this error signal is Lyapunov based [10], with its advantages of robustness, continuous-time implementation and direct adaptation.

Finally, some experimental results obtained from computer simulation (SPICE) in a class-A RF power amplifier are presented.

\section{ADAPTATION UNIT DESIGN}

\section{II.1. General Formulation}

The general scheme of the RF power amplifier linearizer is exposed in figure 1 .

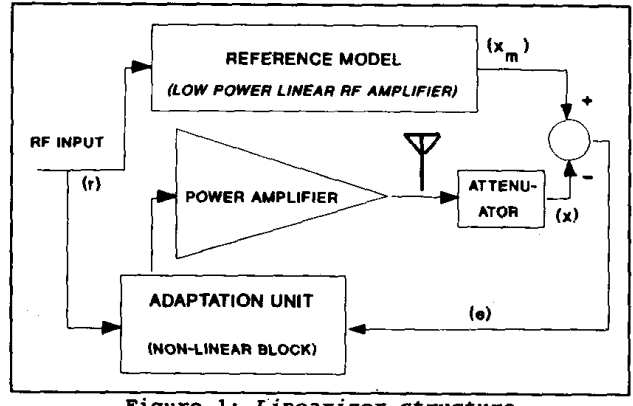

Figure 1: Linearizer structure

lts equivalent design model (MRAS) is shown in figure 2

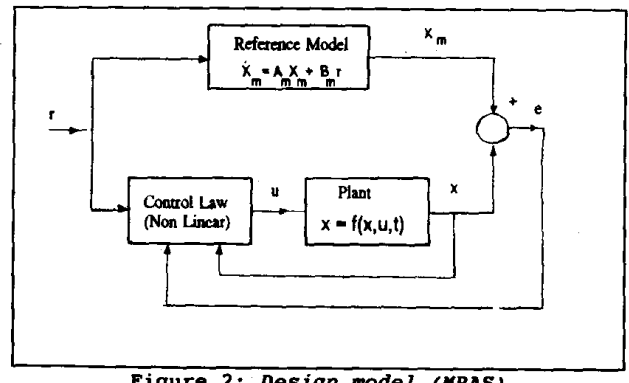

The reference model (controllable) is, in state form:

$$
\dot{x}_{m}=A_{m} x_{m}+B_{m} r
$$

and the RF amplifier ('plant' in figure 2), assumed to be nonlinear and $t$-variant, is:

$$
\dot{x}=f(x, u, t)
$$

The state error vector (e) is defined as:

$$
e=x_{m}-x
$$

with the derivative:

$$
\begin{aligned}
\dot{e} & =\dot{x}_{m}-\dot{x}=A_{m} x_{m}+B_{m} r-f(x, u, t) \\
& =A_{m} e+A_{m} x-f(x, u, t)+B_{m} r
\end{aligned}
$$

The Lyapunov candidate function

$$
V(e)=e^{T} P e
$$

(being $P$ a symmetric, real and positive definite matrix), has the derivative:

$$
\begin{aligned}
& V(e)=\dot{e}^{T} P e+e^{T} P \dot{e} \\
& =\left[A_{m} e+A_{m} x-f(x, u, t)+B_{m} r\right]^{T} P e+e^{T} P\left[A_{m} e+A_{m} x-f(x, u, t)+B_{m} r\right] \\
& =e^{T}\left[A_{m} P+P A_{m}\right] e+\left[x^{T} A_{m}-f^{T}(x, u, t)+r^{T} B_{m}{ }^{T}\right] P e+e^{T} P\left[A_{m} x-f(x, u, t)+B_{m}\right] \\
& =e^{T} P\left[A_{m} P+P A_{m}\right] e+\alpha^{T} P e+e^{T} P \alpha \\
& \left(\alpha^{T} P e\right)^{T}=e^{T} P \alpha=M \\
& \left.\begin{array}{l}
e \in R^{n} \\
P \in R^{n \times m} \\
\alpha \in R^{n}
\end{array}\right\} M \text { bilineal function of } e^{T} \wedge \alpha
\end{aligned}
$$

So,

$$
\begin{gathered}
\dot{V}(e)=e^{T}\left[A_{m}^{T} P+P A_{m}\right] e+M^{T}+M=-e^{T} Q e+2 M \\
-Q=A_{m}^{T} P+P A_{M} \\
M=e^{T} P\left[A_{M} X-f(x, u, t)+B r\right]
\end{gathered}
$$

From Lyapunov theory, if the function $V(e)$ is positive definite and its derivative is negative definite, the system will be asymptotically stable. These general conditions implie:

$$
\begin{gathered}
A_{n}^{T} P+P A_{M}=-Q, \quad \text { Negat.Defin. } \\
M: \text { non positive }
\end{gathered}
$$

\section{I1.2.- Design Example.}

In figure 3 is shown the main scheme of a simple class $A$ power amplifier to be used in the design. There are several options to formulate the model of this plant; we select the phase variables in order to facilitate the formulation. This choice implies the use of a linear observer (Luenberger observer), with a negligible increase in the number of analog components in the final implementation. 


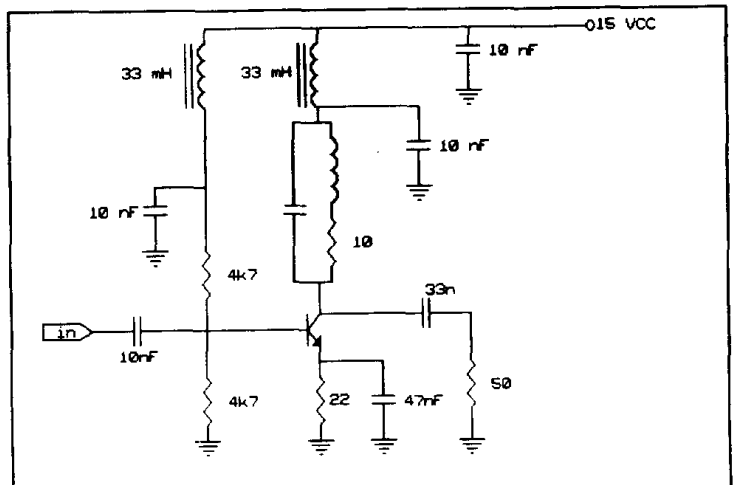

Figure 3: Class A Power Anplifier.

From experimental measurements, the model of the RF power amplifier is (phase variables obtained directly from the amplifier transfer function):

$$
\begin{gathered}
\left(\begin{array}{l}
\dot{x}_{1} \\
\dot{x}_{2}
\end{array}\right)=\left(\begin{array}{cc}
0 & 1 \\
-7,7 \cdot 10^{13} & -19.10^{6}
\end{array}\right)\left(\begin{array}{l}
x_{1} \\
x_{2}
\end{array}\right)+\left(\begin{array}{l}
0 \\
1
\end{array}\right) r \\
y=\left(\begin{array}{ll}
0 & -0,21.10^{9}
\end{array}\right)\left(\begin{array}{l}
x_{1} \\
x_{2}
\end{array}\right)
\end{gathered}
$$

The observer has been designed from the classical Luenberger observer design steps, by choosing its eigenvalues 3 times faster than the observed plant (RF amplifier) ones.

Finally, the reference model has been selected as:

$$
\begin{gathered}
\left(\begin{array}{l}
\dot{x}_{1} \\
\dot{x}_{2}
\end{array}\right)=\left(\begin{array}{cc}
0 & 1 \\
-3,95 \cdot 10^{13} & -3,14 \cdot 10^{6}
\end{array}\right)\left(\begin{array}{l}
x_{1} \\
x_{2}
\end{array}\right)+\left(\begin{array}{l}
0 \\
1
\end{array}\right) r \\
y_{r}=\left(\begin{array}{ll}
0 & -3,14 \cdot 10^{7}
\end{array}\right)\left(\begin{array}{l}
x_{1} \\
x_{2}
\end{array}\right)
\end{gathered}
$$

From the previous models and design equations it is obtained:

$$
M=e^{T} P\left[A_{M} X-f(x, u, t)+B r\right] \leq 0
$$

and, with a linear model of the plant,

$$
f(x, u, t)=A x+B u
$$

it is obtained the equation:

$$
e^{T} P\left[A_{m} x+B_{m} r-A x-B u\right] \leq 0
$$

developing it, the control law $u$ (output of the adaptation unit to the power amplifier in figure 1) is:

$$
u \geq\left|\left(Q-a_{\min }\right) x_{1 p}+\left(R-b_{\min }\right) x_{2 p}+r\right| \cdot \operatorname{sign}\left(e_{1} p_{12}+e_{2} p_{22}\right)
$$

where $e_{1}$ is the output voltage error, $e_{2}=\dot{e}_{1}, x_{1 p}$ the output voltage of the RF amplifier, $k_{2 p}=\dot{x}_{1 p}, a_{\min }$ and $b_{\min }$ are the minimum values of model parameters (initially $\mathrm{a}=-7.710^{13}$ and $b=-1910^{6}$ in equation (9)), and $p_{12}$ and $p_{22}$ are arbitrary values of the positive definite matrix $P$ in equation (5). With our model parameters, the control law (16) becomes:

$$
u=4\left(3.10^{11} x_{1 p}+8.10^{6} x_{2 p}-r\right) \cdot \operatorname{sign}\left(10^{12} e_{1}+e_{2}\right)
$$

being 4 an arbitrary factor.

\section{SIMULATION RESULTS}

The power amplifier of figure 3 has been studied by SPICE simulation in different states of the power transistor. When it is operating in its linear zone, the adaptation loop has no effects. Nevertheless, when it is close the nonlinear zones (cut or saturation), the behaviour of the power amplifier adapted with the proposed structure is significatively improved. The degree of improvement is similar to the obtained with the LINC amplifier. In the proposed structure the analog circuitry adjustment is not a problem. This circuitry consists of the reference model (4 small signal transistor and passive components), the linear observer (4 small signal transistors and passive elements), and the control law (equation (17): 3 single small signal amplifiers, 2 adders and 1 comparator with 4 PIN diodes).

The following figures show the response of the power amplifier operating near the saturation zone. In figure 4.a. and 4.b. it is compared the behaviour, for a single tone (frequency $f_{0}$ ), of the amplifier working without the adaptation loop and with it.

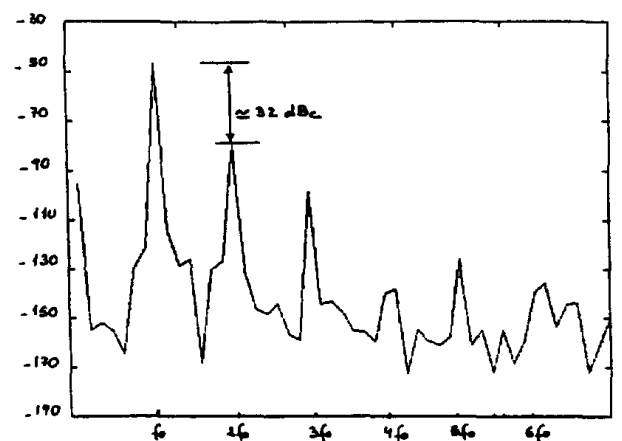

4.a

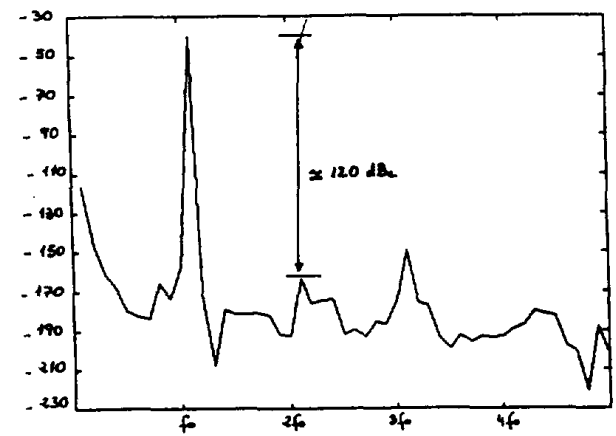

4.b

Figure 4.- Power amplifier response to a single tone $\left(f_{s}\right)$ operating near saturation. a) Without adaptation. b) With adaptation 
The figures 5.a and 5.b. corresponds to the experimentation of the two tone test. The test tones were $1 \%$ far from the nominal frequency $\mathrm{f}_{0}$.

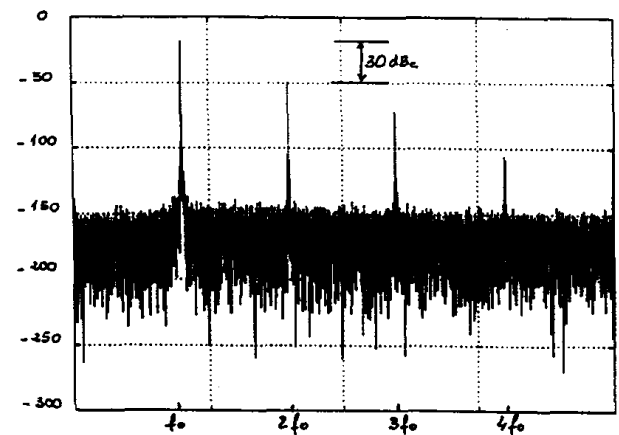

5.a

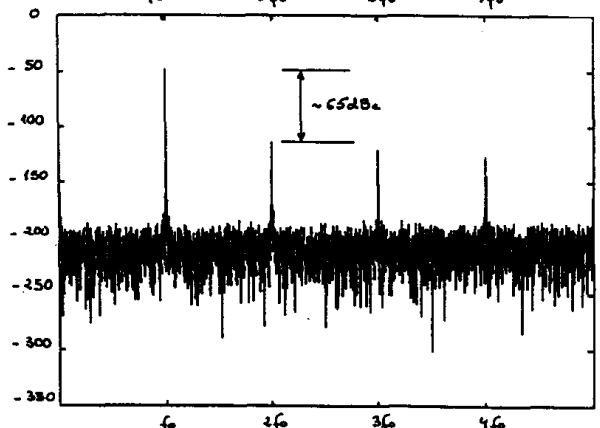

Figure 5.- Two tone response of the power amplifier. a) Without adaptation. b) With adaptation.

In figures 6.a and 6.b it is shown a detail of figures 5.a and 5.b around the nominal frequency $f_{0}$.

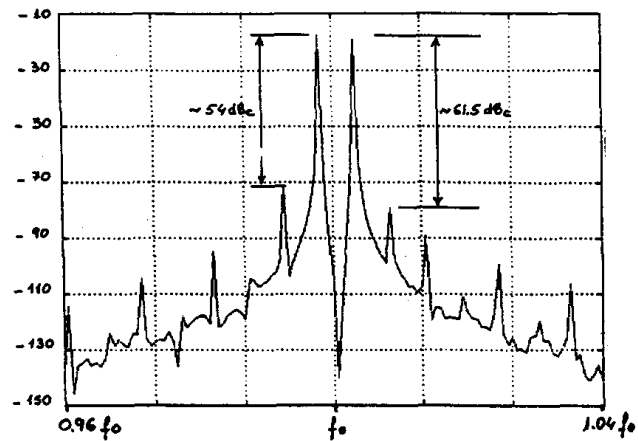

$6 . \mathrm{a}$

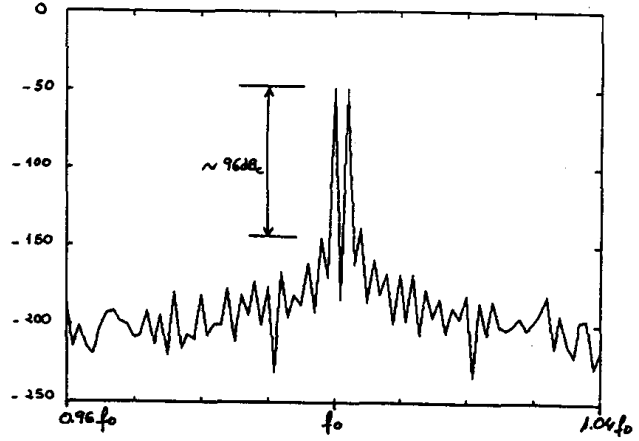

$6 . b$

Figure 6.- Detail of figure 5 around $f_{b}$ a) Without adaptation. b) With adaptation.

The simulation has shown the sensitivity of the results to the comparator quality ('sign' function in equation (17)). By using an ideal comparator these results could be improved

\section{REFERENCES}

[1] Petrovic, V. "Application of Cartesian Feedback to HF SSB Transmitters". Proc. IEE Conf. on $H F$ Communications. 1985

[2] Saleh, A.A.; Salz, J. "Adaptive Linearization of Power Amplification in Digital Radio System". The Bell System Technical Journal. April, 1983.

[3] Wittmarsh, W.J.; Bateman, A; Marvill, J.D. "The Application of Digital Signal Processing in Mobile Radio Transceiver Design". Proceedings of EUSIPCO-90. Barcelona, 1990.

[4] Cox, D.C., Leck, R.P. "Component Signal Separation and Recombination for Linear Amplification with Nonlinear Components". IEEE trans. on Communications. Nov. 1975.

[5] Koh, T. Powers, E.J. (1985). "Second order Volterra Filtering and its Application to Nonlinear System Identification". IEEE trans. on ASSP, vol.33.

[6] Bernardini, A; De Fina, S. "Application of Neural Waveform Predistortion to Experimental TWT Data". Proceedings of Melecon'91. Ljubljana, May 1991.

[7] Landau, I.D. "A Feedback System Approach to Adaptive Filtering". IEEE trans. on Information Theory, March 1984.

[8] Cowan C.F.W, Grant, P.M. "Adaptive Filters". Prentice-Hall, 1985.

[9] Hawksford, M.J., Rezaee, N. "Adaptive mean-squareerror transversal equalizer". IEE proceed. Vol.128., No 5, October 1981.

[10] Landau, Y.D. "Adaptive Control: The Model Reference Approach". Marcel-Dekker, 1979. 